Pacific Journal of Mathematics

THE HASSE-WITT-MATRIX OF SPECIAL PROJECTIVE 


\section{THE HASSE-WITT-MATRIX OF SPECIAL PROJECTIVE VARIETIES}

LEONHARD MILLER

The Hasse-Witt-matrix of a projective hypersurface defined over a perfect field $k$ of characteristic $p$ is studied using an explicit description of the Cartier-operator. We get the following applications. If $L$ is a linear variety of dimension $n+1$ and $X$ a generic hypersurface of degree $d$, which divides $p-1$, then the Frobenius-operator $\mathscr{F}$ on $H^{n}\left(X \cdot L ; \mathcal{O}_{L \cdot Y}\right)$ is invertible.

As another application we prove the invertibility of the HasseWitt-matrix for the generic curve of genus two. We don't study the Frobenius $\mathscr{F}$ directly, but the Cartier-operator [1]. It is wellknown, that for curves Frobenius and Cartier-operator are dual to each other under the duality of the Riemann-Roch theorem. A similar fact is true for higher dimension via Serre duality. We have therefore to extend to the whole "De Rham" ring the description of the Cartier-operator given in [4] for 1-forms. We give this extention in $\S 1$. Diagonal hypersurfaces are studied in $\S 2$ and the invertibility of the Hasse-Witt-matrix is proved, if the degree divides $p-1$. The same theorem for the generic hypersurface follows then from the semicontinuity of the matrix rank. The $\S 3$ is devoted to hyperelliptic curves and is intended as a preparation for a detailed study of curves of genus two.

1. The Cartier-operator of a projective hypersurface. We extend the explicit construction of the Cartier-operator given in [4] to the whole "De Rham" ring, but restrict ourself to projective hypersurfaces.

As an application we show: Let $V$ be a projective hypersurface of dimension $n-1$, defined by a diagonal equation $F(X)=\sum_{i=0}^{n} a_{i} X_{i}^{r}$, $a_{i} \in k$ a perfect field of char $k=p>0, a_{i} \neq 0$. Let $X$ be a linear variety of dimension $t+1$. If $r$ divides $p-1$, then

$$
\mathscr{F}: H^{t}\left(X \cdot V, \mathcal{O}_{X \cdot V}\right) \rightarrow H^{t}\left(X \cdot V, \mathcal{O}_{X \cdot V}\right)
$$

is invertible, $\mathscr{F}$ being the induced Frobenius endomorphism. We have to rely on a technical proposition, which is a collection of some lemmas in [4]. We give first the proposition.

Proposition 1. Let

$$
\psi: k[T] \rightarrow k[T] \quad\left(T=\left(T_{1}, \cdots, T_{n}\right)\right)
$$


be $k p^{-1}$-linear and

$$
\psi\left(T^{\mu}\right)= \begin{cases}T^{\nu} & \text { if } \mu=p \cdot \nu \\ 0 & \text { else }\end{cases}
$$

Then the following holds:

(1) $\psi\left(T_{\mu_{1}} \cdots T_{\mu_{r}} h\right)=T_{\mu_{1}} \cdots T_{\mu_{1}} \bar{h}$, for some $\bar{h} \in k[T]$

(2) Let $D_{\mu}=T_{\mu}\left(\partial / \partial T_{\mu}\right)$ and $D_{\mu} g=0$ for a given $1 \leqq \mu \leqq n$, then $\psi\left(D_{\mu} h \cdot g\right)=0$

(3) Let $D_{\mu} g=0$, then $\psi\left(h^{p-1} D_{\mu} h \cdot g\right)=D_{\mu} h \psi(g)$.

Proof.

(1) By the $p^{-1}$-linearity of $\psi$ we may assume $h$ to be a monomial. The statement follows then directly from the definition of $\psi$.

(2) $\psi$ is $p^{-1}$-linear, so we may assume $h$ to be a monomial

$$
h=T_{1}^{r_{1}} \cdots T_{n}^{r_{n}}, \quad 0 \leqq r_{i} \leqq p-1
$$

(say $\mu=n$ ), then $D_{n} h=r_{n} \cdot h$. If $r_{n}=0$ then (2) is trivially true. So $r_{n} \neq 0$. Again because of $p^{-1}$-linearity we may also assume $g$ to be monomial.

But $D_{n} g=0$, so

$$
g=T_{1}^{v_{1}} \cdots T_{n-1}^{v_{n-1}} \quad 0 \leqq v_{i} \leqq p-1 .
$$

So the exponent of $T_{n}$ in $D_{n} h \cdot g$ is $r_{n}$ and $0<r_{n} \leqq p-1$, therefore not divisible by $p$. The definition of $\psi$ gives

$$
\psi\left(D_{n} h \cdot g\right)=0 \text {. }
$$

(3) We may write

$$
h=f_{0}+f_{1} \cdot T_{\mu}+\cdots+f_{r} \cdot T_{\mu}^{r}, \quad 0 \leqq r \leqq p-1
$$

and

$$
D_{\mu} f_{i}=0 \text {. }
$$

We proceed by induction on $T$. $r=0$ clear. Let $r \geqq 1$, then $h=$ $f+T_{\mu} \bar{h}$ with $D_{\mu} f=0 \operatorname{deg}_{T_{\mu}} \bar{h}<r$. Now

$$
T_{\mu}^{p-1} \bar{h}^{p-1} D_{\mu}\left(T_{\mu} \bar{h}\right)=\left(T_{\mu} \bar{h}\right)^{p}\left(\frac{D_{\mu} T_{\mu}}{T_{\mu}}+\frac{D_{\mu} \bar{h}}{\bar{h}}\right) .
$$

By $p^{-1}$-linearity of $\psi$ and induction assumption for $\bar{h}$ we get

$$
\begin{aligned}
\psi\left(g \cdot T_{\mu}^{p-1} \bar{h}^{p-1} D_{\mu}\left(T_{\mu} \bar{h}\right)\right) & =T_{\mu} \bar{h} \psi(g)+T_{\mu} \psi\left(g \cdot \bar{h}^{p-1} D \bar{h}\right) \\
& =\psi(g)\left(T_{\mu} \bar{h}+T_{\mu} D_{\mu} \bar{h}\right) \\
& =D_{\mu}\left(T_{\mu} \bar{h}\right) \cdot \psi(g)
\end{aligned}
$$


On the other hand

$$
T_{\mu}^{p-1} \bar{h}^{p-1}=(h-f)^{p-1}=h^{p-1}+\frac{\partial P}{\partial h},
$$

where $P$ is a polynomial in $f$ and $h$. We have

$$
D_{\mu}\left(T_{\mu} \bar{h}\right)=D_{\mu}(h-f)=D_{\mu} h .
$$

So

$$
T_{\mu}^{p-1} \bar{h}^{p-1} D_{\mu}\left(T_{\mu} \bar{h}\right)=h^{p-1} D_{\mu} h+D_{\mu} P .
$$

Multiply by $g$ and apply $\psi$, then one gets

$$
D_{\mu} h \cdot \psi(g)=D_{\mu}\left(T_{\mu} \bar{h}\right) \psi(g)=\psi\left(h^{p-1} D_{\mu} h \cdot g\right)+\psi\left(D_{\mu} P \cdot g\right) .
$$

But by (2)

$$
\psi\left(D_{\mu} P \cdot g\right)=0
$$

Let $F\left(X_{0} \cdots X_{n}\right)$ define a absolutely irreducible hypersurface $V / k$ in $\mathscr{P}_{n, k}$ char $k=p>0$. We denote by $f\left(X_{1} \cdots X_{n}\right)$ an affinization of $F$. Let $F_{\mu}=\left(\partial / \partial X_{\mu}\right) F$, similar $f_{\mu} 1 \leqq \mu \leqq n$. We assume $f_{n}$ not to be the zero function on $V$. Let $K=K(V)$ be the function field of $V$. We assume that $K=K^{p}\left(x_{1} \cdots \breve{x}_{j} \cdots x_{n}\right)$ for any index $j$. The $x_{i}$ are the coordinate functions and $\breve{x}_{j}$ means omit $x_{j}$. As a consequence of these assumptions, we have that for a given index $j$ any function $z \in K$ can be represented modulo $F$ by a rational function $G\left(X_{1} \cdots X_{n}\right)$, which is $X_{j}$-constant, i.e. such that $\partial G / \partial X_{j}=0$. Write

$$
F_{i_{1}, \cdots, i_{r, n}}=\left(X_{i_{1}} \cdots X_{i_{r}} \cdot X_{n}\right)^{-1} F \text {. }
$$

DeFinition 1. Let

$$
\psi_{F_{i_{1}}, \cdots, i_{r}, n}=F_{i_{1}, \cdots, i_{r}, n} \circ \psi \circ F_{i_{1}, \cdots, i_{r}, n}^{-1} \cdot
$$

Let $\omega=\sum_{i_{1} \cdots i_{r}} h_{i_{1}, \cdots, i_{r}} \cdot d x_{i_{1}} \wedge \cdots \wedge d x_{i_{r}}$ be $r$-form on $V$. Put

$$
\omega_{i_{1}, \cdots, i_{r}}=\frac{d x_{i_{1}} \wedge \cdots \wedge d x_{i_{r}}}{f_{n}}
$$

Define

$$
C(\omega)=\sum_{i_{1}, \cdots, i_{r}} \psi_{F i_{1}, \cdots, i_{r}, n}\left(h_{i_{1}, \cdots, i_{r}}-f_{n}\right) \omega_{i_{r}, \cdots, i_{r}} .
$$

The definition is justified by the following theorem.

Theorem 1. (1) $C$ is $p^{-1}$-linear

(2) If $\omega=d \varphi$, then $C(\omega)=0$ 
(3) If $\omega=z_{i_{1}}^{p-1} \cdots z_{i_{r}}^{p-1} d z_{i_{1}} \wedge \cdots \wedge d z_{i_{r}}$ then $C(\omega)=d z_{i_{1}} \wedge \cdots \wedge d z_{i_{r}}$. In other words, if one restricts $C$ to $Z_{V / k}^{r}$, the closed forms, then

$$
C: Z_{V / k}^{r} \rightarrow \Omega_{V / k}^{r}
$$

is the Cartier-operator of $V[1]$.

\section{Proof of the theorem.}

(1) The $p^{-1}$-linearity follows from the $p^{-1}$-linearity of $\psi$.

(2) Let $\varphi=\sum_{i_{1}, \cdots, i_{r-1}} \varphi_{i_{1}, \cdots, i_{r-1}} d x_{i_{1}} \wedge \cdots \wedge d x_{i_{r-1}}$ be a $(r-1)$-form, then

$$
d \varphi=\sum_{j} \sum_{i_{1}, \ldots, i_{r}-1} \frac{\partial}{\partial x_{j}}\left(\varphi_{i_{1}, \ldots, i_{r-1}}\right) d x_{j} \wedge d x_{i_{1}} \wedge \cdots \wedge d x_{i_{r-1}}
$$

To simplify the notation we put for the moment

$$
\varphi_{i_{1}, \cdots, i_{r-1}}=\widetilde{\varphi}
$$

and

$$
F_{j_{1} i_{1}, \cdots, i_{r-1}, n}=\widetilde{F} \text {. }
$$

To compute $C(d \varphi)$ we have to compute

$$
\varphi_{\widetilde{F}}\left(\frac{\partial}{\partial x_{j}} \tilde{\varphi} \cdot f_{n}\right)
$$

for every system $\left(j, i, \cdots, i_{r-1}\right)$.

Now remembering the definition of $\psi^{\tilde{F}}$ we have to show

$$
\psi\left(F^{p-1} D_{n} F X_{i_{1}} \cdots X_{i_{r-1}} D_{j} \varphi\right)=0
$$

in order to get $C(d \varphi)=0$.

We have to use the above proposition. We apply first (3) and then (2) and get:

$$
\psi\left(F^{p-1} D_{n} F X_{i_{1}} \cdots X_{i r-1} D_{j} \varphi\right)=D_{n} F \psi\left(X_{i_{1}} \cdots X_{i_{r}-1} D_{j} \varphi\right)=0 .
$$

Remark, that we assume $j \neq\left(i_{1}, \cdots, i_{r-1}\right)$ otherwise

$$
d x_{j} \wedge d x_{i_{1}} \wedge \cdots \wedge d x_{i_{r-1}}=0 .
$$

That shows $C(d \varphi)=0$

(3) Let $\omega=z_{i_{1}}^{p-1} \cdots z_{i_{r}}^{p-1} d z_{i_{1}} \wedge \cdots \wedge d z_{i_{r}}$.

We have 


$$
\begin{aligned}
d z_{i_{1}} \wedge \cdots \wedge d z_{i_{r}} & =\sum_{j_{1} \cdots j_{r}} D_{j_{1}} z_{i_{1}} \cdots D_{j_{r}} z_{i_{r}} \frac{d x_{j_{1}} \wedge \cdots \wedge d x_{j_{r}}}{x_{j_{1}} \cdots x_{j_{r}}} \\
D_{j} & =x_{j} \frac{\partial}{\partial x_{j}}
\end{aligned}
$$

To Compute $C(\omega)$, we have to work out

$$
\begin{gathered}
U=\psi\left(F^{p-1} D_{n} F \cdot Z_{i_{1}}^{p-1} \cdot D_{j_{1}} Z_{i_{1}} \cdots Z_{i_{r}}^{p-1} D_{j_{r}} Z_{i_{r}}\right) \text { modulo } F \\
Z_{j} \bmod F=z_{j}
\end{gathered}
$$

We apply several times (3) of the propositition and get

$$
U \equiv D_{n} F D_{j_{1}} Z_{i_{r}} \cdots D_{j_{r}} Z_{i_{r}} \bmod (F)
$$

Therefore

$$
\begin{aligned}
C(\omega) & =\sum_{j_{r} j_{r}} D_{n} f D_{j_{1}} z_{i_{1}} \cdots D_{j_{r}} z_{i_{r}} \frac{d x_{j_{1}} \wedge \cdots \wedge d x_{j_{r}}}{x_{n} f_{n} x_{j_{1}} \cdots x_{j_{r}}} \\
& =d z_{i} \wedge \cdots \wedge d z_{i_{r}}
\end{aligned}
$$

All forms of highest degree $n-1$ are closed. We use the fact, that $H^{\circ}\left(V, \Omega^{n-1}\right)$ has a basis of the following form

$$
\omega_{u}=x_{1}^{u_{1}} \cdots x_{n}^{u} \omega_{0}
$$

where

$$
\begin{gathered}
\omega_{0}=\frac{d x_{1} \wedge \cdots \wedge d x_{n-1}}{x_{1} \cdots x_{n} f_{n}} \\
\sum_{i=1}^{n} u_{i} \leqq r ; r=\operatorname{deg} V \text { and } 1 \leqq u_{i}
\end{gathered}
$$

Recall $x_{i}=X_{i} / X_{0}$ are coordinate functions on $V$ and of the affinization of $F, f_{n}=\partial f / \partial x_{n}$.

We get the important corollary to the theorem.

COROLlaRy 1. Let $A_{u, v}$ be the matrix of the Cartier-operator on $H\left(V, \Omega^{n-1}\right)$ with respect to the above basis $\omega_{u}$. Then

$$
\begin{aligned}
& A_{u, v}=\text { coefficient of } X^{v} \text { in } \psi\left(F^{p-1} \cdot X^{u}\right) \\
& X^{u}=X_{0}^{u_{0}} \cdots X_{n}^{u_{n}}, \quad \sum_{i=0}^{n} u_{i}=\sum_{i=0}^{n} v_{i}=r \\
& \begin{array}{l}
1 \leqq u_{i} \\
1 \leqq v_{i}
\end{array} \text { for } \quad i=1 \cdots n \text {. }
\end{aligned}
$$


Proof. By definition

$$
\begin{aligned}
C\left(\omega_{u}\right) & =\psi_{F_{1} \cdots n}\left(x_{1}^{u_{1}^{-1}} \cdots x_{n}^{u^{-1}}\right) \frac{d x_{1} \wedge \cdots \wedge d x_{n-1}}{f_{n}} \\
& =\psi\left(f^{p-1} \cdot x^{u}\right) \omega_{0} .
\end{aligned}
$$

Now recall

$$
\begin{aligned}
& \psi\left(f^{p-1} \cdot x^{u}\right)=\psi\left(\frac{F^{p-1} X_{0}^{u_{0}} \cdots X_{r}^{u} r}{X_{0}^{p r}}\right) \bmod F \\
& \sum_{i=0}^{n} u_{i}=r, \quad 1 \leqq u_{i}, \quad i=1 \cdots n .
\end{aligned}
$$

If $A_{u, v}$ is the coefficient of $X^{v}$ in $\psi\left(F^{p-1} \cdot X^{u}\right)$.

Then

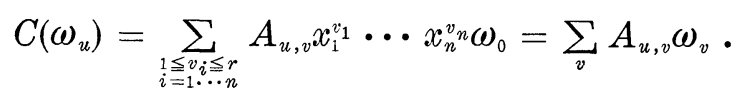

Notice

$$
\sum_{i=0}^{n} u_{i}=\sum_{i=0}^{n} v_{i}=r, \quad 1 \leqq u_{i}, 1 \leqq v_{i}, i=1 \cdots n
$$

REMARK. We have now on explicit description for the Cartieroperator on $H^{0}\left(V, \Omega_{V / k}^{n-1}\right)$. We can use Serre duality $H^{0}\left(V, \Omega_{V / k}^{n-1}\right)^{\vee} \cong$ $H^{n-1}\left(V, \mathscr{O}_{U}\right)$. Under this duality $\check{C}$ is the Frobenius $\mathscr{F}$ on $H^{n-1}\left(V, \mathscr{O}_{V}\right)$. We have therefore also an explicit description for $\mathscr{F}$.

2. The Cartier-operator of a diagonal hypersurface. Let $F(X)=\sum_{i=0}^{n} a_{i} X_{i}^{r}$ define a "generic" hypersurface. To compute the Cartier-operator, by the preceding discussion we have to analyse

$$
\psi\left(F^{p-1} X^{u}\right) \quad\left(\sum_{i=0}^{n} u_{i}=r, \quad u_{i}>0\right)
$$

Let us adapt the following notation:

$$
\begin{gathered}
\rho^{!}=\rho_{0}^{!} \cdots \rho_{n}^{!}, \quad a^{\rho}=\prod_{i=0}^{n} a_{i}^{\rho_{i}}, \quad X^{u+1}=\prod_{i=0}^{n} X_{i}^{u_{i}+1}, \\
|u|=\sum_{i=0}^{n} u_{i}, \quad u>0 \Leftrightarrow u_{i}>0 \quad(i=0 \cdots n) .
\end{gathered}
$$

THEOREM 2. Let

$$
\operatorname{char} k=p>0, F(X)=\sum_{i=0}^{n} a_{i} X_{i}^{r}, \quad \prod_{i=0}^{n} a_{i} \neq 0 \in k
$$

$V / k$ is defined by $F$. Suppose $r$ divides $p-1$. Then the Cartieroperator 


$$
C: H^{\circ}\left(V, \Omega_{V / k}^{n-1}\right) \rightarrow H^{\circ}\left(V, \Omega_{V / k}^{n-11}\right)
$$

is invertible.

Proof.

$$
F^{p-1}=\sum_{|m|=p-1} \frac{(p-1) !}{m !} a^{m} X^{r m}
$$

Using $p^{-1}$-linearity of $\psi$ we get

$$
\psi\left(F^{p-1} X^{u}\right)=\sum_{|m|=p-1} \frac{-1}{m !} \bar{a}^{m} \psi\left(X^{r m+u}\right)=\sum_{|m|=p-1} \frac{-1}{m !} \bar{a}^{m} X^{v}
$$

We put $\bar{a}=a^{1 / p}$, and $r m+u=p v$. Notice if $u>0$ and $|u|=r$, then also $v>0$ and $|v|=r$. If we write

$$
\psi\left(F^{p-1} X^{u}\right)=\sum_{\substack{|v|=r \\ v>0}} A_{u, v} X^{v}
$$

then we have

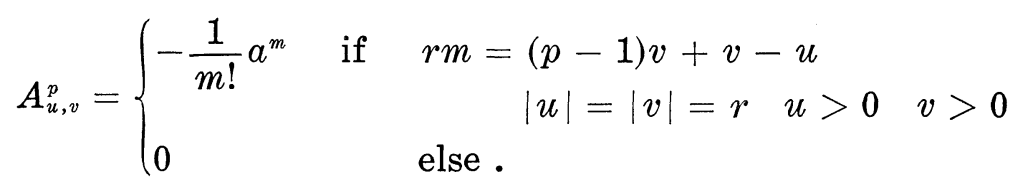

Let us now assume:

$$
p-1=r \cdot s
$$

If $r$ divides $v-u$ put $v-u=r \cdot E(u, v)$ then

$$
A_{u, v}^{p}= \begin{cases}-\frac{1}{m !} a^{m} & \text { if } \quad r \mid v-u \text { and } m=s v+E(u, v) \\ 0 & \text { else . }\end{cases}
$$

We fix now a total ordering of $u, v$. Let us order the $n$-tuples $\left(u_{1} \cdots u_{n}\right)$ resp $\left(v_{1} \cdots v_{n}\right)$ lexicographically and put

$$
u_{0}=r-\sum_{i=1}^{n} u_{i} \quad \text { resp. } \quad v_{0}=r-\sum_{i=1}^{n} v_{i}
$$

$v<u$ means now, that either $v_{1}<u_{1}$ or $v_{i}=u_{i}$ for $i=1 \cdots j-1$ but $v_{j}<u_{j}$. If any case, if $v<u$, then $v_{j}<u_{j}$ for some $j$. We claim if $v<u$, the $A_{u, v}=0$.

Case 1. $r$ does not divide $u-v$, then $A_{u, v}=0$.

Case 2. $r$ divides $u-v$. Now if $v<u$ then for some $j u_{j}-v_{j}>0$ 
and $r$ divides $u_{i}-v_{j}$. But $r \geqq u_{j}$ and $v_{j} \geqq 1$, so $r-1 \geqq u_{j}-v_{j}$, therefore $r$ cannot divide $u_{j}-v_{j}$. This contradiction shows, if $v<u$, then $A_{u, v}=0 . A_{u, v}$ is therefore a triangle matrix.

What is the diagonal?

$$
A_{u, u}^{p}=-\frac{1}{m !} a^{m}
$$

with $m=s \cdot u$. Therefore

$$
\left(\operatorname{det} A_{u, v}\right)^{p}=\prod_{u}\left(-\frac{1}{(s u) !}\right) a^{s \Sigma u} \neq 0
$$

COROLLARY 2. The assumptions are the same as in the theorem. Then

$$
\mathscr{F}: H^{n-1}\left(V, \mathscr{O}_{V}\right) \rightarrow H^{n-1}\left(V, \mathscr{O}_{V}\right) \quad(\mathscr{F} \text { is the Frobenius morphism) }
$$

is invertible.

Proof. Clear by Serre duality and the fact that $\check{C}=\mathscr{F}$.

The Cartier-operator of $W \cdot H$. The differential operator $C$ as given in Definition 1 on $\Omega^{1}$ is by $p^{-1}$-linearity completely determined on $\Omega^{1}$ by its value on $\omega=h \cdot d x$, where $x$ runs through a set of coordinate functions.

We have $C(\omega)=x^{-1} \psi(x h) d x$, that notation is only intrinsic, if $d \omega=0$, because $\psi$ depends on the coordinate system. If we choose a different coordinate system, then we get in general a different operator; but for $\omega$ with $d \omega=0$, we get the same, namely the Cartieroperator.

That fact can be exploited in the following way. Suppose

$$
W=\left\{x_{1}=x_{2} \cdots=x_{t}=0\right\} \cap H .
$$

We write now $C_{H}$ resp. $C_{W}$ for the the operators. The above definition shows $\bigoplus_{i=1}^{t} K d x_{i}$ is stable under $C_{H}$. But by the property of $\psi$, $\psi\left(X_{i} H\right)=X_{i} \bar{H}$ for some $\bar{H}$, we have for

$$
\begin{aligned}
\omega & =x_{i} h d x_{j} \quad i \neq j \quad i, j \text { arbitrary } \\
C_{H}(\omega) & =x_{i} \bar{h} d x_{j} .
\end{aligned}
$$

Let $\mathfrak{U}=\left\{x_{1} \cdots x_{t}\right\}$, then $\mathfrak{N} \Omega_{H / k}^{1} \oplus \bigoplus_{i=1}^{t} \mathcal{O}_{H} d x_{i}$ is stable under $C_{H}$. By the exact sequence

$$
0 \rightarrow \mathfrak{Q} \Omega_{H / k}^{1}+\bigoplus_{i=1}^{t} \bigcirc_{H} d x_{i} \rightarrow \Omega_{H / k}^{1} \rightarrow \Omega_{W / k}^{1} \rightarrow 0
$$

$C_{H}$ induces an operator $C_{W}$ on $\Omega_{W / k}^{1} . C_{W}$ has again the properties 
(1) $C_{W}$ is $p^{-1}$-linear

(2) $C_{W}(d h)=0$

(3) $C_{W}\left(h^{p-1} d h\right)=d h$.

If we restrict $C_{W}$ to the closed forms on $W$, then $C_{W}$ is the Cartieroperator.

Let now $L$ be an arbitrary linear variety. After a suitable coordinate change we may assume $L$ is the intersection of some coordinate hyperplanes. $W=L \cdot H$ has then the above shape.

Let us assume that the hypersurface $H$ has a diagonal defining equation of degree $d$ diving $p-1, p=$ char $k$. Then the above Theorem 1 shows that $C_{W}$ is semisimple on $Z_{W / k}^{1}$. In the same way as before we can extend $C_{W}$ to any $\Omega_{W / k}^{r}$, in particular to $\Omega_{W / k}^{m}$, where $m=\operatorname{dim} W$. As result of this discussion we get:

THEOREM 3. If $L$ is a linear variety of dimension $m+1$, then there exists a hypersurface $H$ of degree $d$, which divides $p-1$, such that

$$
\mathscr{F}: H^{m}\left(L \cdot H, \mathscr{O}_{L \cdot H}\right) \rightarrow H^{m}\left(L \cdot H, \mathscr{O}_{L \cdot H}\right)
$$

is invertible.

3. The Cartier-operator of plane curves. For curves the explicit description of the Cartier-operator is of special interest if one wants to study, how the Cartier-operator varies with the moduli of the curve. Unfortunately one is restricted to plane curves, because the above explicit form of the Cartier-operator is available only for hypersurfaces.

If one specializes the above results to plane curves, one has to assume, that the curve is singularity free.

The space $W=$ \{homogenous forms of degree $d-3\}$ is for nonsingular curves $V$ of degree $d$ isomorphic to $H^{\circ}\left(V, \Omega_{V / k}^{1}\right)$ under

$$
\begin{aligned}
W & \simeq H^{0}\left(V, \Omega_{V / k}^{1}\right) \\
P(X) & \rightarrow P(x) \omega_{0}
\end{aligned}
$$

where the coordinate functions are given by

$$
x=X_{1} / X_{0}, \quad y=X_{2} / X_{0} \bmod F,
$$

$F$ being the defining equation for $V$ and $f(x, y)$ the affinization, $f_{y}$ denotes $\partial f / \partial y$. With that notation $\omega_{0}=d x / f_{y}$.

But it is important to know, that one can give a similar description also for singular curves. Then $W$ is the space of $P(X)$, which define the "adjoint" curves to $V$. These are those curves, which cut out at least the "double point divisor". 
To give an explicit basis depends on nature of the singularities.

Hyperelliptic curves: Let $p=$ char $k>2$.

For a detailed study of the Hasse-Witt-matrix of hyperelliptic curves one needs the explicit Cartier-operator with respect to various "normal forms".

Let the hyperelliptic $V$ be given by $y^{2}=f(x), \operatorname{deg} f(x)=2 g+1$ and such that $f(x)$ has no multiple roots. $V$ has a singularity at "infinity". One could apply the above method and work out the adjoint curves in order to get a basis for $H^{0}\left(V, \Omega_{V / k}^{1}\right)$. But we have already a basis, namely if $\omega=d x / y$ then $\left\{x^{i} \omega \mid i=0 \cdots g-1\right\}$ form a basis.

We specialize the results of $\S 2$ and get from Corollary 1 as matrix for the Cartier-operator with respect to the above basis (let us put $p-1 / 2=m)$ :

$$
A_{u, v}=\text { coefficient of } x^{v+1} \text { in } \psi\left(f(x)^{m} x^{u+1}\right) \quad 0 \leqq u \leqq g-1 .
$$

Legendre form: We assume now the defining equation in Legendre form.

$$
f(x)=x(x-1) \prod_{i=1}^{r}\left(x-\lambda_{i}\right) \quad \begin{aligned}
& r=2 g-1 \\
& \lambda_{i} \neq \lambda_{j} \neq 0,1 .
\end{aligned}
$$

Notation: Let

$$
\begin{aligned}
|\rho| & =\rho_{1}+\cdots+\rho_{r} \\
\lambda^{\rho} & =\lambda_{1}^{\rho_{1}} \cdots \lambda_{r}^{\rho_{n}} .
\end{aligned}
$$

The permutation group of $r$ elements $S_{r}$ operates on the monomials

$$
\lambda^{\rho} \rightarrow \lambda^{-(\rho)}, \pi \in S_{r}
$$

Let $G_{\rho}$ be the fix group of $\lambda^{m-\rho}$ and $G^{(\rho)}=S_{r} / G_{\rho}$. Let

$$
H^{\langle\rho\rangle}(\lambda)=\sum_{\pi \in G(\rho)} \lambda^{m-\tau(\rho)} \text {. }
$$

Apparently

$$
H^{(\rho)}=H^{\overline{(\rho)}}, \quad \text { iff } \quad \bar{\rho}=\bar{\pi}(\rho) .
$$

We may therefore assume

$$
0 \leqq \rho_{1} \leqq \rho_{2} \leqq \rho_{r} \leqq m .
$$

For given

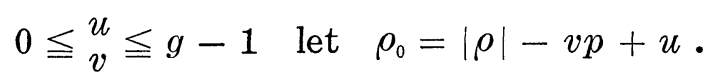


Put

$$
a_{u, v}^{(\rho)}=(-1)^{u+v+m}\left(\begin{array}{c}
m \\
\rho_{0}
\end{array}\right) \cdots\left(\begin{array}{c}
m \\
\rho_{r}
\end{array}\right)
$$

and

$$
A_{u, v}^{p}=\sum_{\rho} a_{u, v}^{(\rho)} H^{(\rho)}(\lambda) \quad 0 \leqq \begin{aligned}
& u \\
& v
\end{aligned} g-1, r=2 g-1
$$

the summation condition being:

$$
\begin{gathered}
0 \leqq \rho_{1} \leqq \cdots \leqq \rho_{r} \leqq m, \quad \rho_{0}=|\rho|-v p+u, \quad 0 \leqq \rho_{0} \leqq m \\
v p-u+m \geqq|\rho| \geqq v p-u .
\end{gathered}
$$

We state as a proposition

Proposition 2. Let be $A_{u, v}, 0 \leqq \underset{v}{u} \leqq g-1$, as defined above, and $\omega=d x / y$, then

$$
C\left(x^{u} \omega\right)=\sum_{0 \leqq v \leqq g-1} A_{u, v} x^{v} \omega
$$

is the Cartier-operator.

Applications: We want to investigate, when the Cartier-operator is invertible. It seems that an answer to that question, without any restrictions is not available. It is therefore worthwhile to have various methods even in special cases. ${ }^{1}$

We restrict ourself to genus 2, although the method could be applied to higher genus, but the calculations would be very easy. Let $p>2$ and $g=2$

$$
\text { i.e. } y^{2}=x(x-1)\left(x-\lambda_{1}\right)\left(x-\lambda_{2}\right)\left(x-\lambda_{3}\right), \quad \lambda_{i} \neq \lambda_{j} \neq 0,1 \quad i \neq j .
$$

The notation is the same as above.

$H^{(\rho)}(\lambda)$ is homogeneous in the $\lambda$ 's of degree $3 m-|\rho|, m=(p-1) / 2$. We have

$$
\begin{aligned}
& A_{u, v}^{p}=\sum_{0 \leqq \rho_{0} \leqq \rho_{1} \leqq \rho_{2} \leqq \rho_{3} \leqq m} a_{u, v}^{(\rho)} H^{(\rho)}(\lambda) \quad 0 \leqq v \leqq 1 \\
& \rho_{0}=|\rho|-v p+u \quad v p-u \leqq|\rho| \leqq v p-u+m \text {. }
\end{aligned}
$$

We want to know of $A_{u, v}^{p}$, what the forms of lowest homogeneous degree in the $\lambda$ 's are. We have to give $|\rho|$ the maximal possible value.

We use the shorthands

$\overline{1 \text { Added in proof: }}$ We settled this question in the meantime, see [6]. 


$$
\left(\begin{array}{c}
m \\
\rho
\end{array}\right)=\prod_{i=1}^{3}\left(\begin{array}{c}
m \\
\rho_{i}
\end{array}\right)
$$

and $D(u, v)=$ degree of the lowest homogeneous term in $A_{u, v}^{p}$. In the list below is $\rho_{0}=\max |\rho|-v p+u$.

\begin{tabular}{c|c|c|c}
$(u, v)$ & $\max |\rho|$ & $\rho_{0}$ & $D(u, v)$ \\
\hline$(0,0)$ & $m$ & $m$ & $p-1$ \\
$(0,1)$ & $3 m$ & $m-1$ & 0 \\
$(1,0)$ & $m-1$ & $m$ & $p$ \\
$(1,1)$ & $3 m$ & $m$ & 0
\end{tabular}.

We get therefore:

$$
\begin{aligned}
& A_{0,0}^{p} A_{1,1}^{p}=\text { terms of degree } p-1+\text { higher terms } \\
& A_{0,1}^{p} A_{1,0}^{p}=\text { terms of degree } p+\text { higher terms } .
\end{aligned}
$$

The lowest degree term $L$ in $\operatorname{det}\left(A_{u, v}\right)^{p}$ is given by

$$
\begin{aligned}
L= & m \sum\left(\begin{array}{c}
m \\
\rho
\end{array}\right) H^{(\rho)}(\lambda) \\
& \rho_{1}+\rho_{2}+\rho_{3}=m \\
& 0 \leqq \rho_{1} \leqq \rho_{2} \leqq \rho_{3} .
\end{aligned}
$$

Notice, if $\rho \neq \bar{\rho}$, then $H^{(\rho)}$ and $H^{(\bar{\rho})}$ have no monomial in common. Therefore $L$ is not the zero polynomial. We are able to specialize the variables $\left(\lambda_{1}, \lambda_{2}, \lambda_{3}\right)$ in the algebraic closure of $k$, such that $\operatorname{det}\left(A_{u, v}\right) \neq 0$. In other words, there exist curves of genus two with invertible Cartier-operator.

We do not know, what the smallest finite field is, over which such a curve exists.

REMARK. For large $p$ we could push through a similar discussion for higher genus. We omit that, because there is a more elegant method for large $p$ by Lubin (unpublished). Let $y^{2}=x^{2 g+1}+a x^{g+1}+x$. The claim is, that for large $p$ (depending on $g$ ) and variable $a$ the Hasse-Witt-matrix of that curve is a permutation matrix.

\section{REFERENCES}

1. P. Cartier, Questions de rationalité des diviseurs en géometrie algébrique, Bull. Soc. Math. France, 86 (1958). 177-251.

2. H. Hasse, Existenz separabler zyklischer unverzweigter Erweiterungskörper vom Primzahlgrade p über elliptischen Funktionenkörpern der Charakteristik p, J. f. r. a. Math., 172 (1934), 77-85.

3. H. Hasse and E. Witt, Zyklische unverzweigte Erweiterungskörper vom Primzahlgrade $p$ über einem algebraischen Funktionenkörper der Characteristik $p$. Monatshefte 
Math. Phys., 43 (1936), 477-493.

4. L. Miller, Über die Punkte der Ordnung p auf einer Jacobischen k-Varietät, char $k$ $=p>0$. Nachr. Akad. Wiss. Göttingen, II. Math.-Phys. Kl. (1969), Nr. 2, 9-23.

5. — Elementarer Beweis eines Satzes von $H$. Hasse über die Punkte der Ordnung $p$ auf einer elliptischen $k$-Kurve, $p=$ char $k$, Nachr. Akad. Wiss. Göttingen, II. Math.-Phys. Kl. (1969), Nr. 1, 1-8.

6. - Curves with invertible Hasse-Witt-matrix (appears in Math. Annalen)

Received June 28, 1971. This research was supported partially by the Ohio State University and partially by NSF-grant GP-20833.

OHio State University 



\section{PACIFIC JOURNAL OF MATHEMATICS}

\section{EDITORS}

\author{
H. SAMELSON \\ Stanford University \\ Stanford, California 94305 \\ C. R. HOBBY \\ University of Washington \\ Seattle, Washington 98105
}

\author{
J. DugundJI \\ Department of Mathematics \\ University of Southern California \\ Los Angeles, California 90007
}

\author{
RICHARD ARENS \\ University of California \\ Los Angeles, California 90024
}

\section{ASSOCIATE EDITORS}
E. F. BECKENBACH
B. H. NeumanN
F. WOLF
K. YoSHIDA

\section{SUPPORTING INSTITUTIONS}

\author{
UNIVERSITY OF BRITISH COLUMBIA \\ CALIFORNIA INSTITUTE OF TECHNOLOGY \\ UNIVERSITY OF CALIFORNIA \\ MONTANA STATE UNIVERSITY \\ UNIVERSITY OF NEVADA \\ NEW MEXICO STATE UNIVERSITY \\ OREGON STATE UNIVERSITY \\ UNIVERSITY OF OREGON \\ OSAKA UNIVERSITY
}

\author{
UNIVERSITY OF SOUTHERN CALIFORNIA \\ STANFORD UNIVERSITY \\ UNIVERSITY OF TOKYO \\ UNIVERSITY OF UTAH \\ WASHINGTON STATE UNIVERSITY \\ UNIVERSITY OF WASHINGTON \\ $* * * *$
$*$
AMERICAN MATHEMATICAL SOCIETY
NAVAL WEAPONS CENTER
}

The Supporting Institutions listed above contribute to the cost of publication of this Journal, but they are not owners or publishers and have no responsibility for its content or policies.

Mathematical papers intended for publication in the Pacific Journal of Mathematics should be in typed form or offset-reproduced, (not dittoed), double spaced with large margins. Underline Greek letters in red, German in green, and script in blue. The first paragraph or two must be capable of being used separately as a synopsis of the entire paper. The editorial "we" must not be used in the synopsis, and items of the bibliography should not be cited there unless absolutely necessary, in which case they must be identified by author and Journal, rather than by item number. Manuscripts, in duplicate if possible, may be sent to any one of the four editors. Please classify according to the scheme of Math. Rev. Index to Vol. 39. All other communications to the editors should be addressed to the managing editor, Richard Arens, University of California, Los Angeles, California, 90024.

50 reprints are provided free for each article; additional copies may be obtained at cost in multiples of 50 .

The Pacific Journal of Mathematics is published monthly. Effective with Volume 16 the price per volume (3 numbers) is $\$ 8.00$; single issues, $\$ 3.00$. Special price for current issues to individual faculty members of supporting institutions and to individual members of the American Mathematical Society: $\$ 4.00$ per volume; single issues $\$ 1.50$. Back numbers are available.

Subscriptions, orders for back numbers, and changes of address should be sent to Pacific Journal of Mathematics, 103 Highland Boulevard, Berkeley, California, 94708.

PUBLISHED BY PACIFIC JOURNAL OF MATHEMATICS, A NON-PROFIT CORPORATION

Printed at Kokusai Bunken Insatsusha (International Academic Printing Co., Ltd.), 270, 3-chome Totsuka-cho, Shinjuku-ku, Tokyo 160, Japan. 


\section{Pacific Journal of Mathematics}

\section{Vol. 43, No. 2 \\ April, 1972}

Arne P. Baartz and Gary Glenn Miller, Souslin's conjecture as a problem on the real line....................................... 277

Joseph Barback, On solutions in the regressive isols ............... 283

Barry H. Dayton, Homotopy and algebraic K-theory ................ 297

William Richard Derrick, Weighted convergence in length ............ 307

M. V. Deshpande and N. E. Joshi, Collectively compact and semi-compact sets of linear operators in topological vector spaces ............. 317

Samuel Ebenstein, Some $H^{p}$ spaces which are uncomplemented in $L^{p} \ldots . .327$

David Fremlin, On the completion of locally solid vector lattices ......... 341

Herbert Paul Halpern, Essential central spectrum and range for elements of

a von Neumann algebra............................... 349

G. D. Johnson, Superadditivity intervals and Boas' test ............. 381

Norman Lloyd Johnson, Derivation in infinite planes . . . . . . . . . . . 387

V. M. Klassen, The disappearing closed set property .............. 403

B. Kuttner and B. N. Sahney, On the absolute matrix summability of Fourier series ........................................... 407

George Maxwell, Algebras of normal matrices................... 421

Kelly Denis McKennon, Multipliers of type $(p, p) \ldots \ldots \ldots \ldots \ldots \ldots . . \ldots 29$

James Miller, Sequences of quasi-subordinate functions ............. 437

Leonhard Miller, The Hasse-Witt-matrix of special projective varieties ..... 443

Michael Cannon Mooney, A theorem on bounded analytic functions ...... 457

M. Ann Piech, Differential equations on abstract Wiener space .......... 465

Robert Piziak, Sesquilinear forms in infinite dimensions ............. 475

Muril Lynn Robertson, The equation $y^{\prime}(t)=F(t, y(g(t))) \ldots \ldots \ldots \ldots .483$

Leland Edward Rogers, Continua in which only semi-aposyndetic

subcontinua separate ............................... 493

Linda Preiss Rothschild, Bi-invariant pseudo-local operators on Lie

groups ...................................... 503

Raymond Earl Smithson and L. E. Ward, The fixed point property for

arcwise connected spaces: a correction ...................... 511

Linda Ruth Sons, Zeros of sums of series with Hadamard gaps .......... 515

Arne Stray, Interpolation sets for uniform algebras............... 525

Alessandro Figà-Talamanca and John Frederick Price, Applications of random Fourier series over compact groups to Fourier multipliers .. 